\title{
ROBOT SOCIAL COMO ASISTENTE DE COCINA
}

\author{
Jaime Gómez-Jiménez, Sara Carrasco-Martínez, M. A. Quispe-Flores, Javier Sevilla-Salcedo, \\ Fernando Alonso-Martín, Miguel A. Salichs \\ RoboticsLab, Departamento de Ingeniería de Sistemas y Automática, \\ Universidad Carlos III de Madrid
}

100383149@alumnos.uc3m.es, \{sacarras, mquispe, jasevill\}@pa.uc3m.es, \{famartin, salichs\}@ing.uc3m.es

\section{Resumen}

En el presente trabajo se describe una aplicación real para los robots sociales del departamento de automática en la Universidad Carlos III, para dotar a los robots Mini y Milow de la capacidad de convertirse en asistentes de cocina. La asistencia consiste en una interacción multimodal mediante una interfaz gráfica y diálogos, ofreciendo las indicaciones al usuario de los pasos a realizar en el momento de elaborar un plato culinario. De tal manera, este trabajo mejora la capacidad de los robots añadiendo otra funcionalidad.

Palabras clave: Robótica social; Accesibilidad; Android; Cocina; Recetas.

\section{INTRODUCCIÓN}

La evolución tecnológica que está ocurriendo actualmente ha dado lugar a un gran impulso para la robótica en diversos ámbitos, siendo uno de ellos la robótica social. Esta disciplina permite una interacción fluida con el usuario mediante el uso de diferentes medios de comunicación como los gestos, diálogos entre ambos y expresiones propias del robot [1]. De esta manera, la robótica social se estima que sea un recurso clave para todas aquellas personas que necesiten compañía y así mantener una conversación o entretenerse con robots sociales. Asimismo, a medida que las personas llegan a cierta edad, es más frecuente que haya problemas al realizar las tareas básicas mientras están cocinando, debido al deterioro cognitivo y la pérdida de memoria. Por ejemplo, pueden olvidarse de realizar un paso, o bien no añadir todos los ingredientes necesarios [2].

En el presente artículo se describe el desarrollo de una aplicación, también llamada habilidad, con el objetivo de asistir al usuario durante la elaboración de platos culinarios, estando implementada en los robots sociales Mini y Milow. Se pretende crear una aplicación que cubra todas las necesidades de accesibilidad que permita su uso a las personas mayores en el momento de cocinar. Varios estudios han demostrado que la preparación de recetas culinarias favorece a una mejora de la salud tanto mental como física y, por tanto, es una tarea fundamental para hacer en el día a día [3].

$\mathrm{El}$ artículo tiene la siguiente estructura. La Sección 2 investiga los trabajos relacionados con este artículo desde tres perspectivas diferentes: los robots sociales, los robots de cocina y las aplicaciones para móviles de recetas. Más adelante, en la Sección 3 se muestran los robots donde se implementa el trabajo desarrollado y , posteriormente, la Sección 4 describe la propuesta realizada. Finalmente, la Sección 5 recoge los datos obtenidos en las pruebas realizadas a usuarios y en la Sección 6 se concluyen los resultados.

\section{ESTADO DEL ARTE}

El campo de la robótica ha ido mejorando a lo largo de los años, incorporando nuevas habilidades a los robots. Esas habilidades pueden variar desde la construcción de vehículos en un nivel industrial hasta la asistencia en hospitales o en tareas del hogar, como es el caso de los robots sociales. En esta Sección se estudiará los trabajos relacionados tanto con la robótica social como con la preparación de recetas desde tres perspectivas diferentes: los robots sociales, robots de cocina y aplicaciones para móvil de recetas.

\subsection{ROBOTS SOCIALES}

Aquellos robots destinados a la asistencia de personas o a la terapia, son los robots sociales, y suelen tener apariencias amigables similares a los animales. Estos robots incluyen una gran variedad de habilidades, que abarcan desde aplicaciones de entretenimiento como es el caso de los juegos, pasando por habilidades para ver fotos y escuchar música, hasta aquellas destinadas a realizar ejercicios de estimulación cognitiva. Este conjunto de habilidades permiten al usuario interactuar con el robot para entretenerse en el día a día. Ejemplos de este tipo de robots pueden ser Milow [4], Mini [5] o Pepper [6] entre otros.

\subsection{ROBOTS DE COCINA}

Comer es una actividad esencial para las personas y un robot capaz de elaborar cualquier plato 
de cocina podría aportar valor al usuario, a pesar del reto que supone para el campo de la robótica. Dependiendo del grado implicación en la cocina, se pueden separar en tres categorías. Por una parte, se encuentran los robots que vienen integrados en la propia cocina, como es el caso de la Cocina Moley, capaz de cocinar platos completos sin ayuda del usuario ya que puede acceder a todas las secciones de la cocina [7].

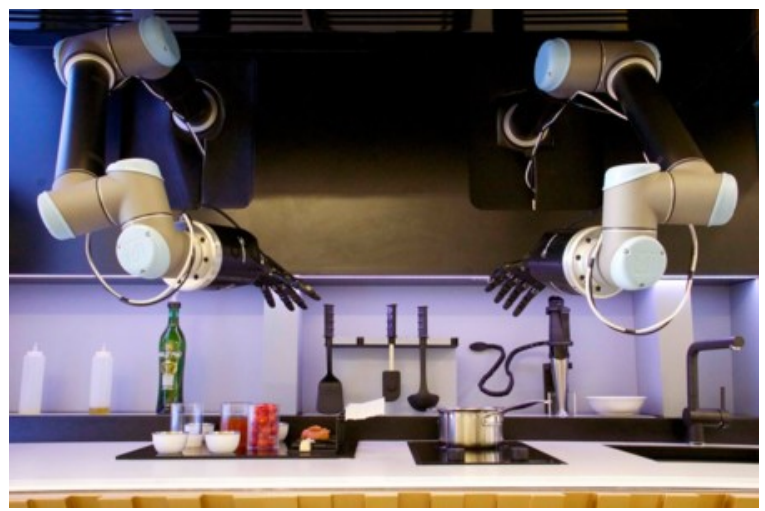

Figura 1: Robot de Cocina Moley [7].

Por otra parte, están los robots que requieren la ayuda del usuario para introducir los ingredientes y seleccionar las acciones que el robot debe realizar, siendo el caso de la Thermomix [8]. Por último, cabe destacar aquellos robots que son únicamente informativos, como Alexa, que contiene la habilidad de cocina ${ }^{1}$ y le permite indicar todos los ingredientes y los pasos necesarios que debe seguir el usuario para la elaboración de las recetas $[9]$.

\subsection{APLICACIONES DE RECETAS}

Actualmente, en el mercado de aplicaciones para dispositivos móviles, existe una gran variedad destinadas al uso y creación de recetas, siguiendo todas el mismo patrón: contienen una lista de recetas organizadas por categorías e incluso algunas tienen la posibilidad de filtrar dichas recetas según los ingredientes que desee el usuario o bien según la dieta que esté siguiendo el mismo.

Ejemplos de aplicaciones de recetas cabe destacar la app Oui Chef, desarrollada con la opción de sugerir los menús de acuerdo a las preferencias del usuario, además de contener una calculadora de calorías para todas sus recetas [10]. Otra aplicación sobresaliente es la de Inverse Cooking, capaz de recrear los ingredientes y los pasos de un plato culinario atendiendo a una foto del mismo. Gracias a su amplia base de datos es posible predecir

\footnotetext{
${ }^{1}$ Sitio Web de la habilidad: https://www . amazon.es/Weblogs-SL-Directo-al-Paladar/dp/ B07 JVD1297
}

los ingredientes disponibles en la foto y generar instrucciones de manera automática [11].

\section{METODOLOGÍA}

Este artículo se centra en la implementación de la habilidad de recetas en los robots Mini y Milow, desarrollados por el Laboratorio de Robótica Social de la Universidad Carlos III de Madrid. Ambos robots contienen los mismos módulos principales de Software, siendo la diferencia más notable entre ambos los cambios en el diseño estético.

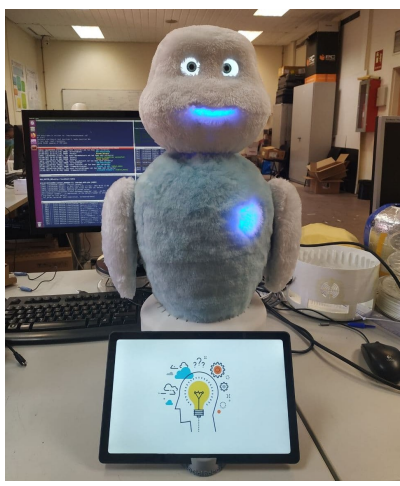

(a) Robot Milow [4].

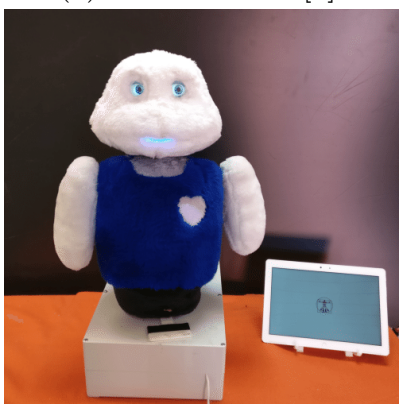

(b) Robot Mini [12].

Figura 2: Robots desarrollados por el RoboticsLab.

\subsection{DESCRIPCIÓN DE LOS ROBOTS SOCIALES UTILIZADOS}

Estos robots están destinados al entretenimiento de los usuarios mediante el uso de habilidades o juegos como el ahorcado, el bingo o bien reproduciendo música y fotos según los gustos del usuario [12]. Asimismo, también contienen ejercicios de estimulación cognitiva para asistir a aquellos que lo necesiten. Para conseguir la interacción entre el usuario y el robot social, dispone de una tableta vinculada que muestra el contenido multimedia [13], además de poder pronunciar frases y reconocer lo que el usuario quiere decir para una comunicación más natural entre ambos.

Los módulos presentes en estos robots y que han 
sido utilizados en el presente proyecto se pueden observar en la Figura 3.

- Sistema de Toma de Decisiones (DMS): permite el control de todas las habilidades por parte del robot de forma autónoma sin necesidad de un "operador", dando lugar a que pueda tomar sus propias decisiones [14].

- Gestor de interacción Humano-Robot (HRI Manager): se encarga de controlar las órdenes recibidas por las habilidades mediante el uso de Acciones Comunicativas, activando los módulos pertinentes. Además, procesa la información procedente de los actuadores y sensores del robot [15].

- Reconocimiento automático de voz (ASR): tiene como objetivo el procesamiento de las indicaciones realizadas por el usuario a través de la voz, enviando el resulado al HRI Manager [16].

- Reproductor de voz (TTS): se encarga de sintetizar el texto enviado por el HRI Manager y reproducirlo en los altavoces, informando al usuario cuando interactúa con el robot [17].

- Gestor de expresividad: aporta mayor vivacidad al robot mediante el movimiento de los motores, el control de los ojos y la activación de luces presentes en el corazón y mejillas [18].

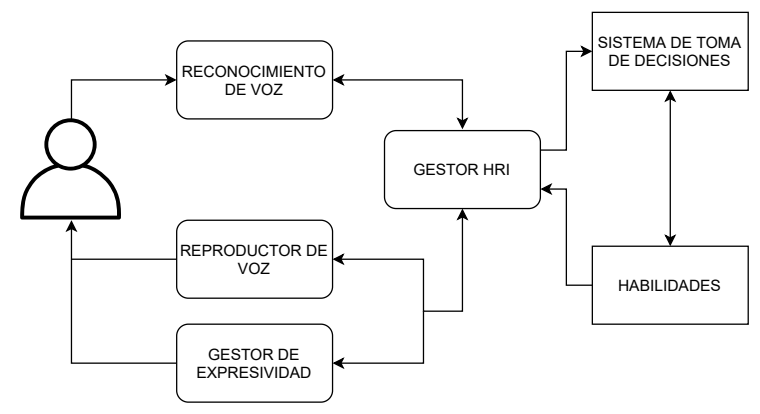

Figura 3: Diagrama de software del robot Milow.

\section{NUESTRA PROPUESTA}

La habilidad de recetas expuesta en este artículo, tiene como objetivo la asistencia de personas mayores a la hora de cocinar, permitiendo la búsqueda y creación de recetas culinarias a través de los robots sociales, utilizando las funcionalidades principales del robot para mantener una comunicación natural con el usuario.

\subsection{PROPIEDADES GENERALES}

El proyecto se divide en tres módulos principales: el robot social, la aplicación de Android [19] y el paquete de ROS (Robot Operating System) [20] llamado recipes_skill.

Para el segundo módulo se ha utilizado el ejemplo de android_tutorial_pubsub procedente del proyecto android core ${ }^{2}$. Este ejemplo proporciona los recursos necesarios para conseguir una conexión de Android con ROS, utilizando una comunicación bidireccional de publicador-suscriptor. De esta manera, es posible el paso de información entre la aplicación de Android y el paquete de ROS, siendo este último el encargado de comunicarse con los módulos presentes en el robot social.

De forma paralela a la ejecución de la aplicación, la habilidad utiliza los actionlibs, un tipo de mensajes de ROS que permite al robot iniciar o parar la habilidad cuando sea necesario a través del DMS.

\subsection{REQUISITOS PREVIOS}

Es necesario tener presentes todos los archivos necesarios en la tableta que poseen los robots, siendo estos la aplicación general, la aplicación de Recetas y el archivo XML para su correcta conexión con el robot que se desee.

Asimismo, es imprescindible que ambas aplicaciones tengan permisos para acceder al almacenamiento del dispositivo y para poder mostrarse sobre otras aplicaciones.

Finalmente, es necesario tener el paquete recipes_skill en el entorno de trabajo del robot a utilizar.

\subsection{DISEÑO ESTÉTICO}

La aplicación muestra la información tanto de forma visual a través de la tableta como por los altavoces del robot indicando las acciones que el usuario puede realizar. En cuanto a la tableta, dispone de tres opciones dentro del menú principal, como se puede ver en la Figura 4.

La opción principal y más útil es la de "Explorar", permitiendo a los usuarios buscar nuevas recetas en una lista e incluso filtrarlas según el nombre o el ingrediente que desee utilizar (ver Fig. 5). Una vez elegida la receta deseada, se muestra un resumen de la receta, incluyendo los ingredientes, los pasos y el tiempo que requiere para su elaboración. En esta pantalla, se puede añadir la receta a favoritos, o bien, empezar a cocinar directamente.

\footnotetext{
${ }^{2}$ Proyecto android_tutorial_pubsub: https : //github.com/rosjava/android_core/tree/ kinetic/android_tutorial_pubsub
} 


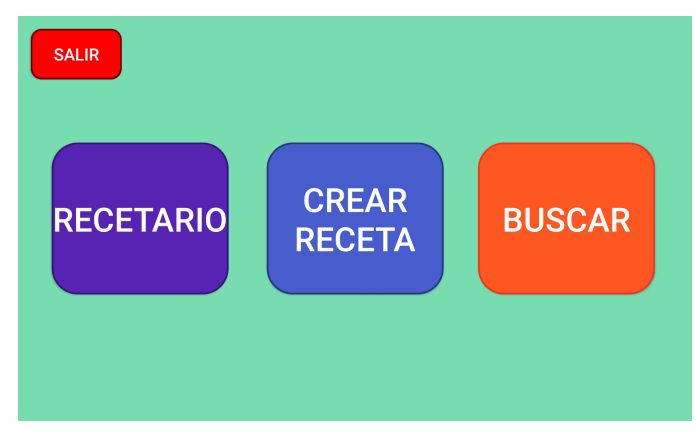

Figura 4: Diseño gráfico de la pantalla principal.

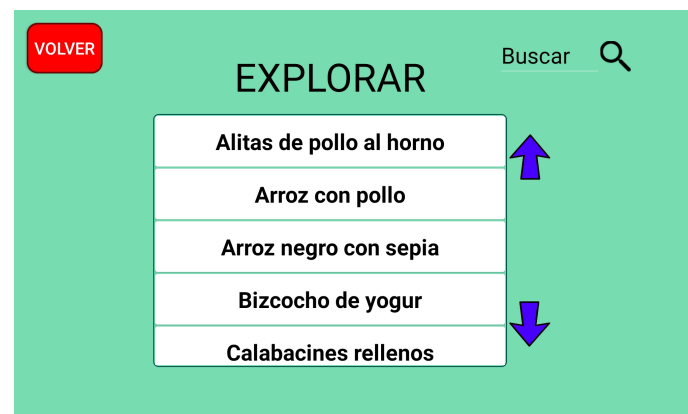

Figura 5: Diseño gráfico del menú de exploración.

Si la elección del usuario es empezar la receta, la aplicación muestra primeramente la lista de los ingredientes que son necesarios junto a las cantidades de cada uno y, cuando el usuario los tiene preparados, el robot va indicando cada paso que se debe realizar para poder cocinar el plato elegido. Esta parte puede incluir un temporizador ajustado a cada paso como se puede observar en la Figura 6 , donde el círculo va disminuyendo a medida que pasa el tiempo.

En cuanto a la opción de "Nueva receta", la aplicación ofrece la posibilidad de crear una receta totalmente personalizada, empezando por añadir el título, seguido por incluir todos los ingredientes uno a uno y terminando por la parte de los pasos. Esta pantalla permite añadir acciones junto a sus ingredientes además de especificar la temperatura o un temporizador si es necesario.

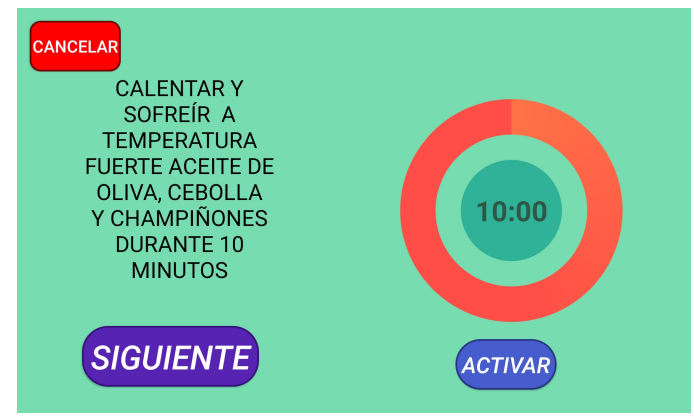

Figura 6: Diseño gráfico del menú de cada paso.
Finalmente, la opción de "Mis recetas" dispone de dos listas de recetas, las cuales contienen las recetas creadas por el usuario y aquellas añadidas por favoritos desde la sección de "Explorar". La única diferencia entre estas dos listas es que la primera posibilita la edición de recetas, ya sea añadiendo o eliminando pasos e ingredientes.

Adicionalmente, se han incorporado varios accesorios para mejorar el diseño estético del robot. Como se puede apreciar en la Figura 7, Milow está agarrando una cuchara de madera y tiene puesto un gorro de chef. Para conseguir lo primero, se ha diseñado una pieza de sujeción para el brazo, acompañada de otra parte para la cuchara, permitiendo que se puedan diseñar más piezas distintas y así poder personalizar el robot con otros accesorios como un micrófono. Estas implementaciones permiten al usuario personalizar el robot según lo que vaya a hacer con él, aportando mayor interacción con el mismo.

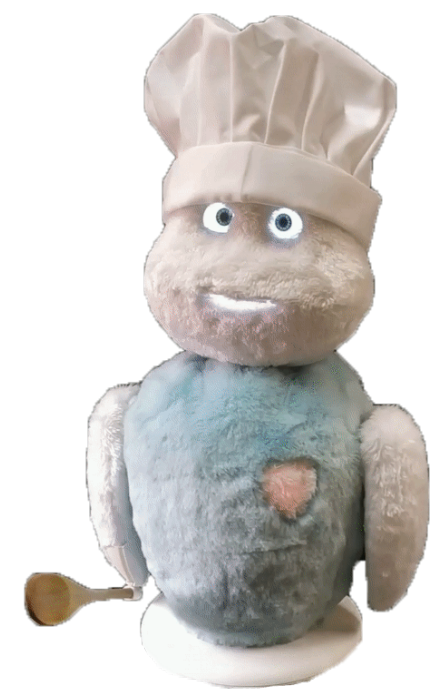

Figura 7: Accesorios añadidos al robot Milow.

\subsection{IMPLEMENTACIÓN}

Para la correcta implementación y funcionamiento de la habilidad de recetas en los robots sociales, todos los módulos deben comunicarse entre sí manteniendo la prioridad de los mismos. Dentro del proyecto, la aplicación en Android se encarga de manejar toda la lógica de los menús que se muestran en la tableta, enviando la información mediante mensajes de ROS a la habilidad (recipes_skill) presente en el robot. De tal forma, cada vez que el usuario realiza una opción en la tableta, envía la información al robot, que a su vez se comunica con el HRI Manager según lo que desee hacer. Una vez se comunica con el HRI Manager, este activa el ASR, TTS o los Gestos dependiendo del contenido del mensaje y, cuando termina de 
pronunciar una frase o de escuchar al usuario, la habilidad recibe el resultado.

Por tanto, existe una comunicación bidireccional entre los módulos, mientras que en paralelo el DMS puede parar o cancelar la actividad cuando el usuario toque el brazo del robot o cuando este lo estime oportuno.

\section{EVALUACIÓN}

Para comprobar el uso de la aplicación en usuarios, se ha realizado una encuesta a personas de todas las edades acerca del estilo y la accesibilidad de la habilidad de recetas. Los usuarios indican que prefieren un estilo con botones, evitando el uso de elementos como barras para deslizar a los cuales no están tan acostumbrados. Asimismo, el hecho de que se pueda navegar tanto por voz como por la pantalla de la tableta, aporta a los usuarios una sensación de comodidad a la hora de moverse por la aplicación. Además, se ha preguntado a los encuestados acerca de las recetas que les gustaría incluir en el robot, permitiendo que las recetas predefinidas mostradas en el menú de "Explorar" sean aquellas que prefieren la mayoría de personas.

Como conclusión a las pruebas realizadas en usuarios, el resultado ha sido razonablemente positivo, con una puntuación de $\mathbf{4 . 3 4}$ sobre $\mathbf{5}$ (ver Fig. 8).

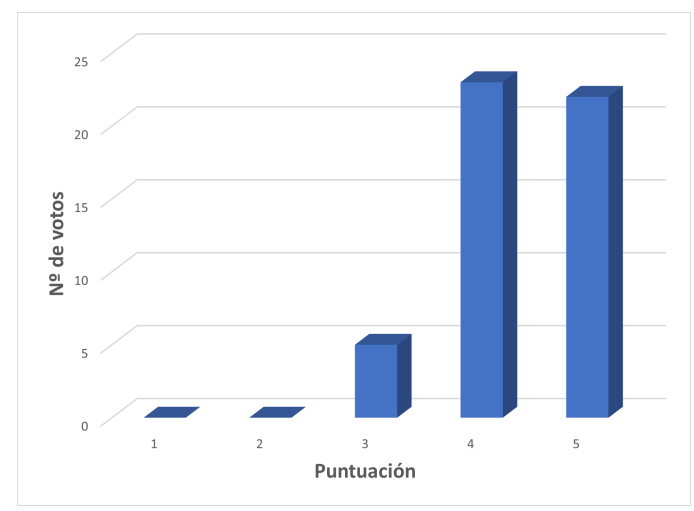

Figura 8: Puntuación obtenida en la encuesta a los usuarios.

\section{CONCLUSIONES}

Este artículo se centra en el desarrollo de una habilidad de recetas para poder convertir a los robots sociales Mini y Milow en asistentes de cocina. Gracias al desarrollo tanto de una interfaz gráfica como de una navegación por voz, los usuarios han mencionado que el robot aporta mayor facilidad a la hora de utilizar la aplicación. Por otro lado, se puede concluir que las encuestas realizadas han sido de mucha utilidad, ayudando a mejorar aspectos de diseño para así evitar confusión durante su uso y también proporcionando una lista de recetas que prefieren la mayoría de usuarios para poder incluirla en la aplicación por defecto.

Asimismo, debido a la creación de la aplicación a través del entorno de Android, se ha conseguido crear una interfaz con mejoras visuales respecto a otras habilidades de los robots, además de poder incluir más información en cada menú.

\section{English summary}

\section{SOCIAL ROBOT AS COOKING ASSISTANT}

\begin{abstract}
In this paper, it is described a real application for the social robots developed in the automation department of the University Carlos III, to endow the robots Milow and Mini with the capacity of becoming cooking assistants. The assistance consists of a multimodal interaction through a graphic interface and dialogues, offering indications to the user of the cooking steps when it comes to preparing a culinary dish. In this way, this project improves the capacity of the robot adding another functionality.
\end{abstract}

Keywords: Social robotics; Accessibility; Android ; Cooking; Recipes.

\section{Referencias}

[1] Brian R. Duffy and Colm Rooney and G. M. P. (Greg M. P.) O'Hare and Ruadhan O'Donoghue, «What is a social robot?»en, sep. de 1999.

[2] D. Dawson, G. Hendershot y J. Fulton, «Aging in the Eighties: Functional Limitations of Individuals Age 65 Years and Over: (605432007-001),» en, American Psychological Association, inf. téc., 1987, Type: dataset. [En línea]. Disponible en: http://doi. apa . org/get-pe-doi . cfm?doi=10 . 1037/ e605432007-001 (Acceso: 22-06-2021). 
[3] N. Farmer, K. Touchton-Leonard y A. Ross, «Psychosocial Benefits of Cooking Interventions: A Systematic Review,» en, Health Education 85 Behavior, vol. 45, n. ${ }^{\circ} 2$, pp. 167-180, abr. de 2018, Publisher: SAGE Publications Inc. [En línea]. Disponible en: https : / / doi . org / 10 . 1177 / 1090198117736352 (Acceso: 22-06-2021).

[4] C. Gómez, C. Ursúa, J. Montoya, CastroGonzález, F. Alonso Martín, M. Malfaz, M. Maroto Gómez y M. Salichs, «Desarrollo de una versión de bajo coste del robot social Mini,» ago. de 2019, pp. 718-725.

[5] E. Salichs, E. Fernández-Rodicio, J. Castillo, Castro-González, M. Malfaz y M. Salichs, «A Social Robot Assisting in Cognitive Stimulation Therapy,» en, jun. de 2018, pp. 344-347.

[6] Z. A. barakeh, S. alkork, A. S. Karar, S. Said y T. Beyrouthy, «Pepper Humanoid Robot as a Service Robot: a Customer Approach,» en 2019 3rd International Conference on Bio-engineering for Smart Technologies (BioSMART), abr. de 2019, pp. 1-4.

[7] M. Bollini, S. Tellex, T. Thompson, N. Roy y D. Rus, «Interpreting and Executing Recipes with a Cooking Robot,» en Experimental Robotics: The 13th International Symposium on Experimental Robotics, ép. Springer Tracts in Advanced Robotics, J. P. Desai, G. Dudek, O. Khatib y V. Kumar, eds., Heidelberg: Springer International Publishing, 2013, pp. 481-495. [En línea]. Disponible en: https : //doi .org/10 . 1007/978-3-31900065-7_33 (Acceso: 28-03-2021).

[8] A. Fries, A.-W. Bergmeister y M. Spindler, «Thermomix by Vorwerk - A New Way of Cooking,» en Fallstudienkompendium Hidden Champions: Innovationen für den Weltmarkt, J.-P. Büchler, ed., Wiesbaden: Springer Fachmedien, 2018, pp. 73-90. [En línea]. Disponible en: https : / / doi . org / 10 . 1007 / $978-3-658-17829-1$ _ 5 (Acceso: 17-05-2021).

[9] E. Lee, G. Vesonder y E. Wendel, «Eldercare Robotics - Alexa,» en 2020 11th IEEE Annual Ubiquitous Computing, Electronics Mobile Communication Conference (UEMCON), oct. de 2020, pp. 0820-0825.

[10] E. Darwish y M. A. Aziz, «Android Cooking Application,» 2018, Accepted: 202002-16T09:54:37Z. [En línea]. Disponible en: https://repository.najah.edu/handle/ 20.500.11888/14792 (Acceso: 19-05-2021).

[11] A. Salvador, M. Drozdzal, X. Giro-i Nieto y A. Romero, «Inverse Cooking: Recipe Generation from Food Images,» arXiv:1812.06164 [cs], jun. de 2019, arXiv: 1812.06164 version: 2. [En línea]. Disponible en: http : / / arxiv .org/abs / 1812.06164 (Acceso: 19-05-2021).

[12] E. Velazquez-Navarro, S. Gonzalez-Diaz, F. Alonso-Martın, J. C. Castillo, M. Malfaz y M. A. Salichs, «El robot social Mini como plataforma para el desarrollo de juegos de interacción multimodales,» 2019. 
[13] S. Marques, J. Castillo, F. Alonso Martín, M. Maroto Gómez, J. J. Gamboa y M. Salichs, «Interfaces táctiles para Interacción Humano-Robot,» sep. de 2017.

[14] M. Maroto Gómez, Castro González, J. C. Castillo Montoya, M. Malfaz Vázquez y M. Salichs Sánchez-Caballero, «A Bio-inspired Motivational Decision Making System for Social Robots Based on the Perception of the User,» ago. de 2018, Accepted: 2019-0208T09:26:21Z Publisher: MDPI. [En línea]. Disponible en: https : / / e - archivo . uc3m . es / handle / 10016 / 28021 (Acceso: 21-06-2021).

[15] E. Fernández-Rodicio, Castro-González, F. Alonso-Martín, M. Maroto-Gómez y M. Salichs, «Modelling Multimodal Dialogues for Social Robots Using Communicative Acts,» eng, Sensors (Basel, Switzerland), vol. 20, n. ${ }^{\circ}$ 12, E3440, jun. de 2020.

[16] F. Alonso Martín y M. Salichs, «Integration of a Voice Recognition System in a Social Robot,» Cybernetics and Systems, vol. 42, pp. 215-245, mayo de 2011.

[17] F. Alonso-Martín, M. Malfaz, CastroGonzález, J. Castillo y M. Salichs, «Online Evaluation of Text to Speech Systems for Three Social Robots,» en, nov. de 2019, pp. 155-164.

[18] E. Fernández-Rodicio, S. Marques y M. Salichs, «Gesture Management in the Robot Mini,» mar. de 2019.
[19] A. Kharisma, «What is Android?» ht tp://developer. android. com/guide ..., [En línea]. Disponible en: https : / / www . academia.edu/2537177/What_is_Android (Acceso: 21-05-2021).

[20] M. Quigley, B. Gerkey y W. D. Smart, Programming Robots with ROS: A Practical Introduction to the Robot Operating System. .'Reilly Media, Inc.", nov. de 2015, GoogleBooks-ID: Hnz5CgAAQBAJ.

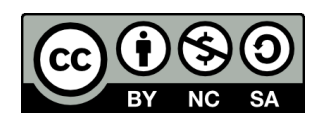

(C) 2021 by the authors. Submitted for possible open access publication under the terms and conditions of the Creative Commons Attribution CC BY-NC-SA 4.0 license (https://creativecommons.org/licenses/by-ncsa/4.0/deed.es). 